\title{
Disturbed maturation of oligodendrocyte progenitors in lipopolysaccharide-induced hypomyelination in cultured forebrain slices of neonatal rats
}

\author{
Jong-Wan Kim ${ }^{1}$, Kun Song Lee ${ }^{2}$, Young Pyo Chang ${ }^{2}$ \\ ${ }^{1}$ Institute of Tissue Regeneration Engineering (ITREN), Dankook University, Cheonan, ${ }^{2}$ Department of Paediatrics, College \\ of Medicine, Dankook University, Cheonan, South Korea
}

\begin{abstract}
Introduction: This study was performed to determine whether the disturbed maturation of oligodendrocyte (OL) progenitors might be related to lipopolysaccharide (LPS)-induced hypomyelination.

Material and methods: We created organotypic cultures of forebrain slices from neonatal rats and explored the morphological changes of glial cells expressing tumour necrosis factor $\alpha$ (TNF- $\alpha$ ) following LPS exposure.

Results: We observed marked activation of glial fibrillary acidic protein-positive astrocytes and OX42-positive microglia co-labelled with TNF- $\alpha$ four days following LPS exposure. Our results further demonstrated a reduced expression of 04-positive and O1-positive OL progenitors; moreover, we found that their morphologies were suggestive of degeneration (e.g., scanty, rounded bodies with short, fragmented processes and/or cytoplasmic condensation). At seven days following LPS exposure, astrocytes and microglia were still co-labelled for TNF- $\alpha$; however, the expression of O4-positive and O1-positive cells somewhat increased compared to the number observed at 4 days; despite remaining undifferentiated and exhibiting immature morphologies, the cells were likely indicative of regeneration. In contrast, 04-positive and 01-positive cells in controls were well-differentiated, displaying round, thick cell bodies and long, branching processes.

Conclusions: In conclusion, maturation arrest and/or under-differentiation of OL progenitors commonly occur during regeneration: they may underlie the degeneration and consequent hypomyelination occurring late after injury, or apoptosis during the acute stage post-injury. Microglia and astrocytes expressing TNF- $\alpha$ may also contribute to later myelination failure.
\end{abstract}

Key words: maturation arrest, oligodendrocyte progenitor, hypomyelination, glial cells, lipopolysaccharide, proinflammatory cytokine.

\section{Introduction}

Pre-oligodendrocytes (preOL) [33] are the main target cells of white matter injury (WMI) of premature brains $[2,3,5,33,34]$. It has been proposed that the degeneration or death of oligodendrocyte (OL) progenitors and the consequent deficit of preOL may cause myelination failure in WMI of the premature brain [2,33]; the underlying mechanism, however, has yet to be elucidated. Prior research hints at the mechanism's possible complexity: though it is well known that surviving preOL rapidly regenerate and increase in number to compensate for the 
death of preOL $[2,4,6,7,9,28]$, morphologies of these newly regenerated preOL remain in an undifferentiated state $[7,28]$. These findings suggest that - in addition to preOL degeneration or death - the arrest of $\mathrm{OL}$ progenitor maturation during post-injury repair may be related to hypomyelination in a chronic state of WMI in the premature brain $[6,7,9,28]$. This hypothesis has been supported by human and experimental studies showing that the failure of preOL to differentiate into mature OLs with diffuse astrogliosis may be significantly related to myelination failure in chronic WMI $[4,6,7,9,13,23,26,28]$ High-field magnetic resonance imaging studies have also supported the characterization of preOL maturation arrest and gliosis as common forms of WMI among preterm survivors $[23,26]$.

Astrogliosis and microgliosis are further recognized as prominent pathologic features of WMI in premature infants $[2,23,34]$ : in response to hypoxia-ischemia and inflammation, activated astrocytes and microglia release proinflammatory cytokines particularly tumour necrosis factor $\alpha$ (TNF- $\alpha)$ and, to a lesser extent, interleukin $1 \beta$ (IL-1 $\beta$ ) - that induce OL apoptosis and hypomyelination [2,12,14,18,21,25,35]; under pathologic conditions such as periventricular leukomalacia and multiple sclerosis, activated glial cells release various inflammatory mediators that could induce axonal injury and damage preOLs $[10,11,15,16,19,20,22,27,30]$. These findings are consistent with experimental studies that have revealed the pivotal role of astrocytes and microglia in mediating repair after injury $[22,27]$. Though the impact of WMI in the premature brain on the degeneration and differentiation of glial cells remains poorly understood, recent investigations have made progress: using an organotypic slice culture model of chronic WMI, a study found that myelination failure is related to diffuse white matter astrogliosis, disturbance of OL maturation, and hyaluronan accumulation [9]; inducing spinal cord injury in adult rats, an investigation showed that reactive astrocytes in the glial scar released TNF- $\alpha$ to inhibit the survival of OL progenitors in demyelinating lesions and thus prevented them from differentiating into mature OLs [30].

The 4 successive stages of oligodendrocyte development-early oligodendrocyte progenitors, late preOLs, immature oligodendrocytes, and mature oligodendrocytes - can be defined by the presence of various type-specific surface antigens [24]. The 01 antibody labels immature oligoden- drocytes, whereas the 04 antibody binds to both preOLs and immature oligodendrocytes [24].

In the current study, we investigated the morphology of OL progenitors using organotypic forebrain slice culture of neonatal rats and immunofluorescent staining with $\mathrm{O} 4$ and $\mathrm{O} 1$ antibodies for $\mathrm{OL}$ progenitors, and to determine whether the disturbed maturation of $\mathrm{OL}$ progenitors might be related to lipopolysaccharide (LPS)-induced hypomyelination. We also investigated the role of inflammation in LPS-induced hypomyelination by observing changes in glial cells expressing TNF- $\alpha$.

\section{Material and methods}

\section{Animals}

Sprague-Dawley rats (3-day-old) used in this study were purchased from Joong Ang Lab Animal (Seoul, Korea) and were certified by the vendor to be pathogen-free for all known rodent bacterial, viral, and parasitic pathogens. Rats were housed on a $12: 12 \mathrm{~h}$ light-dark cycle, temperature $\left(22-25^{\circ} \mathrm{C}\right)$ and humidity (40-60\%) controlled room with food and water available ad libitum. The animals used in this experiment were treated in accordance with the National Institutes of Health Guidelines for Animal Research and were approved by Dankook University Institutional Animal Care and Use Committee (IACUC).

\section{Organotypic culture of forebrain slices}

Organotypic cultures of forebrain slices from neonatal rats were obtained using the modified interface culture methods described previously [29]. Briefly, the forebrains of 3-day-old Sprague-Dawley rat pups were carefully removed and placed on a petri dish over ice. They were then coronally cut $1 \mathrm{~mm}$ anterior and $3 \mathrm{~mm}$ posterior to the bregma. The resulting sections were placed in a cold $\left(12-14^{\circ} \mathrm{C}\right)$ modified Krebs-Henseleit solution (cutting solution) containing $\mathrm{NaCl}(120 \mathrm{mM})$, $\mathrm{KCl}(2 \mathrm{mM}), \mathrm{CaCl}_{2}(0.5 \mathrm{mM}), \mathrm{NaHCO}_{3}(26 \mathrm{mM}), \mathrm{MgSO}_{4}$ $(10 \mathrm{mM}), \mathrm{KH}_{2} \mathrm{PO}_{4}(1.18 \mathrm{mM})$, glucose $(11 \mathrm{mM})$, and sucrose $(200 \mathrm{mM})$ : the high quantity of sucrose created a hyperosmolar solution that prevented brain oedema, while the high magnesium-to-calcium ratio precluded neuronal injury from $N$-methyl-d-aspartate (NMDA) receptor activation. The forebrain was then sliced (400 $\mu \mathrm{m}$ thickness) using a Mcllwain tissue chopper (Mickle Laboratory, Guilford, UK). Slices were preincubated in a sucrose-free slicing solution that was oxygenated with $95 \% \mathrm{O}_{2} / 5 \% \mathrm{CO}_{2}$ for at least 
30 minutes. Slices were then carefully separated, selected, and transferred to Millicell culture inserts (Millipore, Bedford, MA, USA). The inserts were placed into six-well plates with $1 \mathrm{ml}$ serum-free Neurobasal ${ }^{\circledR}$ medium (Gibco, Rockville, MD, USA), supplemented with B27 (Gibco), 1 mM L-glutamine, $100 \mathrm{U} / \mathrm{ml}$ penicillin, and $100 \mu \mathrm{g} / \mathrm{ml}$ streptomycin; the culture medium was changed every 1 to 3 days. We added LPS (5 $\mathrm{\mu g} / \mathrm{ml}$; Escherichia coli O111:B4, Sigma, St. Louis, MO, USA) to the culture media on day 3 of the in vitro prep. The slices were exposed to LPS for 24 hours and were maintained for the following 14 days. Cultured slices were divided into two groups: LPS-exposed and control. Sections were also prepared for immunofluorescent staining and western blotting.

\section{Measurement of TNF- $\alpha$ in culture media}

The culture medium of forebrain slices - from both the control and LPS-exposed groups - were serially collected at $0,3,4,5$, and 7 days after LPS exposure. The levels of TNF- $\alpha$ were measured by performing an enzyme-linked immunosorbent assay (ELISA).

\section{Immunofluorescent staining}

We used the following primary antibodies for immunohistochemistry: mouse anti-O4 and anti-O1 antibodies (MAB345 and MAB344, dilution 1 : 100; Millipore, Bedford, MA, USA) for the detection of OL progenitors, goat polyclonal anti-myelin basic protein (MBP, 1 : 100; Santa Cruz Biotechnology, Santa Cruz, CA, USA) for myelin, rabbit anti-glial fibrillary acidic protein (GFAP, 1 : 1000; DAKO, Carpinteria, CA, USA) for astrocytes, mouse anti-OX42 (1 : 200, Millipore) for microglia, and rabbit polyclonal antiTNF- $\alpha$ (1 : 400, Abcam, Cambridge, UK). For immunohistochemistry using antibodies against TNF- $\alpha$, GFAP, and OX42, frozen forebrain slices were sectioned at $10 \mu \mathrm{m}$ thickness 4 and 7 days after LPS exposure. For MBP, sections were obtained 14 days after LPS exposure. The obtained sections were incubated overnight at $4^{\circ} \mathrm{C}$ with primary antibodies against TNF- $\alpha$. After three 10-min washes in phosphate-buffered saline, sections were incubated with secondary antibodies (dilution 1 : 200; Alexa Fluor 555 - anti-mouse IgG, Molecular Probes, Carlsbad, CA, USA) at room temperature for $1 \mathrm{~h}$. To evaluate the source of TNF- $\alpha$, slices labelled with primary anti-TNF- $\alpha$ were double-stained with GFAP and OX42; the sections were then evaluated under fluorescent (Olympus, Tokyo, Japan) and confocal laser (Carl Zeiss, Jena, Germany) microscopes. The number of cells positive for either $\mathrm{O} 4$ or $\mathrm{O} 1$ and GFAP or OX42 were counted at high power field (HPF) in a minimum of six randomly selected white matter fields per slice. Comparisons were made between the LPS-exposed and control groups for each of the six slices.

\section{Western blotting for myelin basic protein}

Cultured forebrain slices were homogenized and centrifuged 14 days after LPS exposure. The supernatant was removed from the centrifuged samples and stored at $-80^{\circ} \mathrm{C}$. Proteins $(50 \mu \mathrm{g} /$ lane $)$ were separated on a 4-20\% gradient polyacrylamide gel under reducing conditions, transferred onto an Immobilon-P transfer membrane (Millipore) using a semidry apparatus (Bio-Rad, Hercules, CA, USA), and incubated overnight at $4^{\circ} \mathrm{C}$ with anti-MBP antibody. Protein bands were subsequently labelled using peroxidase-conjugated goat anti-rabbit secondary antibody (1 : 3000, Jackson Immuno-Research) and Super Signal West Pico Chemiluminescent substrate (Thermo Scientific, Rockford, IL, USA). Glyceraldehyde-3-phosphate dehydrogenase (GAPDH, 1 : 3000, Santa Cruz) was used as a loading control to ensure equal distribution of samples. The intensity of the protein signal was quantified using Multi Gauge V3.0 Image software (Fujifilm Ltd, Tokyo, Japan). Relative densities were thus compared between the LPS-exposed slices $(n=4)$ and controls $(n=4)$.

\section{Statistical analysis}

All statistical analyses were performed using SPSS version 23.0 (SPSS, Chicago, IL). Values were compared between groups using the Student $t$ test and Mann-Whitney $U$ test. $P$-values of less than 0.05 were considered to be statistically significant.

\section{Results \\ ELISA-evaluated TNF- $\alpha$ levels and TNF- $\alpha$ immunofluorescence}

The measurement of TNF- $\alpha$ in culture media by ELISA showed that TNF- $\alpha$ levels peaked 4 days after LPS exposure due to an inflammatory reaction in forebrain slices; the values subsequently decreased. These levels were significantly higher than those 
observed in control slices at both 4 and 5 days after LPS (Fig. 1A). Immunofluorescence yielded similar results: expression of TNF- $\alpha$ in LPS-exposed forebrain slices was elevated 4 days after LPS exposure relative to controls (Fig. 1B, C). The expression of TNF- $\alpha$ in LPS-exposed forebrain slices decreased 7 days after LPS application; however, it remained higher than that observed in control slices (Fig. 1D, E).

\section{Double-staining with anti-TNF- $\alpha$ and anti-GFAP}

Compared to controls, LPS markedly increased the immunofluorescent expression of GFAP-positive astrocytes and TNF- $\alpha$ in forebrain slices at 4 and 7 days after LPS exposure (Fig. 2A-L); many GFAP-positive astrocytes appeared yellowish in colour due to the co-expression of TNF- $\alpha$ (Fig. 2C, I), while few astrocytes appeared to double-label with TNF- $\alpha$ in control slices (Fig. 2F, L). Numbers of these yellowish astrocytes co-expressed with TNF- $\alpha$ were significantly higher at 4 and 7 days after the LPS exposure than those in the controls ( $p<0.05$, Fig. $2 M)$.

\section{Double-staining with anti-TNF- $\alpha$ and anti-OX42}

Compared to control slices, cells positive for OX42 and TNF- $\alpha$ markedly increased at both 4 and 7 days after LPS exposure (Fig. 3A-L). LPS activated OX42-positive microglia, inducing the latter's adoption of various forms with many processes; such microglia were stained yellowish due to the co-expression of TNF- $\alpha$ (Fig. 3A, C, G, I). In contrast, a smaller number of OX42-positive cells with relatively few processes were observed in controls. Moreover, compared to forebrain slices evaluated 4 and 7 days after LPS exposure, control slices sampled the same time points featured fewer instances of cells double-labelled with OX42 and TNF- $\alpha$ (Fig. 3D, F, J, L). However, this difference was only significant at 4 days after LPS exposure $(p<0.05$, Fig. 3M).

\section{Double-staining for anti-TNF- $\alpha$ / anti-GFAP and anti-TNF- $\alpha /$ anti-OX42}

Confocal laser microscopy revealed detailed morphologies of GFAP-positive astrocytes and OX42-positive microglia. GFAP- and OX42-positive cells exhibited many well-differentiated processes and yellow cell bodies due to co-localization with TNF- $\alpha$ at 4 and 7 days following LPS exposure (Fig. 4A, C, E, G); cells from control slices, on the other hand, displayed no co-localization with TNF- $\alpha$ (Fig. 4B, D, F, H).

\section{Immunofluorescent staining for 04 and 01}

The expression of 04- and 01-positive cells in treated forebrain slices appeared significantly decreased relative to controls at four days following LPS exposure (Fig. 5A-D, Q, $p<0.05$ ). HPF showed that 04-positive and 01-positive cells in LPS-exposed forebrain slices had scanty cell bodies with processes that were fewer in number, thinner, more fragmented, and shorter compared to those of control forebrain slices. Some 04-positive and 01-positive cells in LPS-exposed forebrain slices were also seen to have elongated cell bodies with short bipolar or multipolar processes (Fig. 5E, G), suggesting that some cells might have remained in the immature stages of differentiation. The expression of 04-positive and 01-positive cells at 7 days after LPS increased compared to the expression observed after 4 days (Fig. $5 \mathrm{I}, \mathrm{K}$ ), but HPF and magnified images (yellow box) revealed 04-positive cells with elongated cell bodies and relatively short multipolar processes; these results provide further support for the possibility that these cells remained in immature stages of differentiation (Fig. 5M). At 7 days following LPS exposure, the morphology of O1-positive cells appeared typical, rounded and displayed few processes; the observation strongly suggests maturation arrest (Fig. 50). In contrast, O4-positive and O1-positive cells in controls exhibited well-differentiated morphologies: round, thick cell bodies and long, branching processes (Fig. 5F, H, N, P). Magnified images of slices at both 4 and 7 days after LPS exposure revealed more detailed 04-positive and 01-positive cell morphologies; the images allowed us to identify features of degeneration, such as cytoplasmic condensation and fragmented, short processes, as well as immature cells with rounded or elongated cell bodies and few processes. The number of 04-positive and 01-positive cells in LPS-exposed slices was significantly reduced relative to controls 4 days after LPS application ( $p<0.05$; Fig. 5Q, R); though the difference persisted at 7 days, there was no statistically significant difference between the two groups in the 01-positive cells count ( $p>0.05$ in 01; Fig. 5Q, R). We suspect that the lack of significance was likely due to a regeneration-induced increase in the number of 01-positive cells in LPS-exposed slices. 


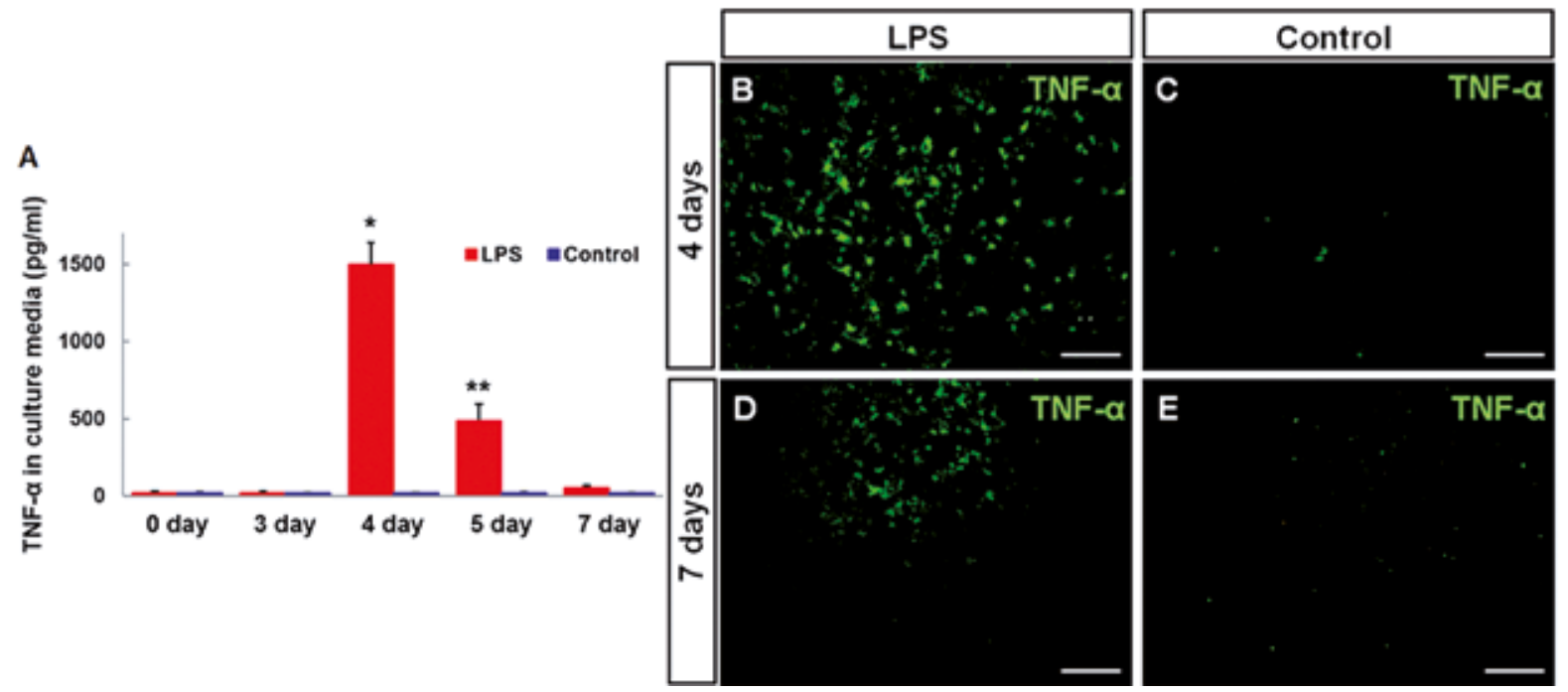

Fig. 1. Measurement of TNF- $\alpha$ in culture media by ELISA, and evaluation of TNF- $\alpha$ expression in forebrain slices via immunofluorescence. ELISA showing that TNF- $\alpha$ levels peaked 4 days after LPS exposure, after which the levels gradually decreased. TNF- $\alpha$ levels were significantly higher at both 4 and 5 days after LPS exposure compared to levels observed in control culture media $\left({ }^{*},{ }^{* *} p<0.05\right)$ (A). Immunofluorescent staining showing that the expression of TNF- $\alpha$ in forebrain slices increased by 4 days after LPS exposure compared to controls (B, C). By 7 days after LPS exposure, the expression of TNF- $\alpha$ had decreased in LPSexposed forebrain slices but was still higher than that of controls (D, E). Scale bars, $200 \mu \mathrm{m}$.

\section{Confocal laser microscopic findings of 04-positive and 01-positive cells}

At 4 days following LPS exposure, confocal laser microscopy revealed degenerative morphological changes in 04-positive and 01-positive cells. These changes included cytoplasmic condensation and fragmented, scanty processes (Fig. 6A, C). Though most 04-positive cells still appeared degenerated at 7 days following LPS exposure, some 04-positive and many 01-positive cells displayed typical round bodies with few short processes; these results suggest a high incidence of under-differentiation (Fig. 6E, G). In contrast, 04- and 01-positive cells in control slices exhibited well-differentiated morphologies, with thick, round cell bodies and long, multipolar processes (Fig. 6B, D, F, H).

\section{Immunofluorescent staining and western blotting for myelin basic protein}

LPS-exposed slices displayed a reduced amount of MBP immunofluorescence compared to controls 14 days after exposure (Fig. 7A, B): where the latter group featured an MBP-associated fluorescent sig- nal extending from subcortical areas to the cortex, the signal in LPS-exposed slices was mostly confined to subcortical areas. Western blot data affirmed the results by also finding that MBP expression was significantly reduced at 14 days after LPS exposure.

\section{Discussion}

Myelination failure in premature brains following chronic WMI is speculated to arise from the degeneration or death of OL progenitors and consequent deficit of preOL $[2,3,5,33]$. However, several recent human and animal studies have elaborated on the hypothesis: though extensive degeneration of preOLs occurs in acute lesions, abnormal differentiation or arrested preOL maturation may contribute to myelination failure in chronic lesions $[2,4,6,7,9,13,26,28]$.

The present study aimed to model the acute phase of WMI; we found that the number of 04-positive and 01-positive cells were markedly reduced in LPS-exposed forebrain sections compared to control forebrain slices at 4 days after exposure. Moreover, O4-positive and 01-positive cells in LPS-exposed tissue exhibited degenerated morphologies, showing cytoplasmic condensation, scanty cell bodies, and 

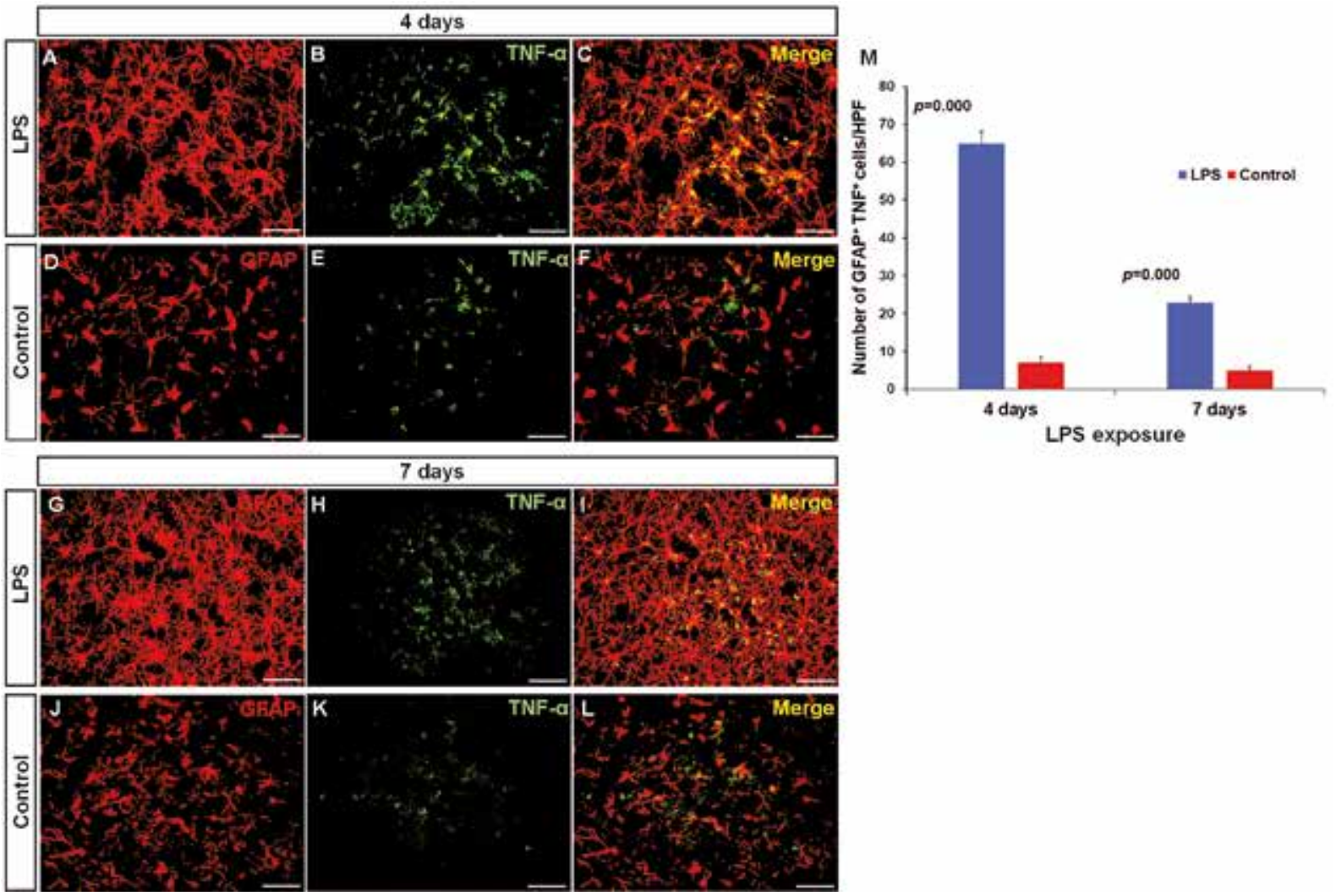

Fig. 2. Double-staining with anti-TNF- $\alpha$ and anti-GFAP. Image showing that the immunofluorescent expression of GFAP and TNF- $\alpha$ in forebrain slices obtained 4 and 7 days after LPS application markedly increased compared to controls (A-L). LPS-exposed GFAP-positive astrocytes (red) appear yellowish in colour due to co-localization with TNF- $\alpha$ (green) (C, I). Images show that few astrocytes are co-localized with TNF- $\alpha$ in controls (F, L). Scale bars, $100 \mu \mathrm{m}$. Numbers of GFAP-positive astrocytes merged with TNF- $\alpha$ per high power field (HPF) in the forebrain slices at 4 and 7 days after the LPS exposure were significantly higher than those in the controls $(p<0.05)(\mathbf{M})$.

thin, fragmented processes. By 7 days following LPS exposure, the number of 04-positive and 01-positive cells had increased; the regeneration of surviving $\mathrm{OL}$ progenitors may explain this change. However, these regenerated $\mathrm{OL}$ progenitors remained morphologically immature: their cell bodies were rounded or elongated, and they exhibited a few short processes. These findings suggest that while surviving OL progenitors tend to regenerate after injury, they remain immature and do not differentiate into mature OL progenitors. We further found that some OL progenitors remained in the early stage of differentiation - a period in which they are resistant to damage in the acute stage of the injury (i.e., 4 days after LPS) $[3,5]$.

Hypoxia-ischemia and infection/inflammation are recognized as important contributors to the WMI of premature brains [14,25]. In addition to having an important role in the perinatal response to brain injury $[11,19,27]$, astrocytes and microglia have also been shown to be involved in the subsequent recovery $[17,22,27]$ : they mediate different aspects of neuronal differentiation, migration, programmed cell death, neurite growth, axon guidance, and synaptic formation [12,19,32]. In many neurological diseases (including white matter disorders), activated microglia and astrocytes release a large number of inflammatory mediators, such as highly reactive oxygen/ nitrogen species and proinflammatory cytokines [1]. Cytokines released from activated microglia and astrocytes - particularly TNF- $\alpha$ - mediate the death of OL progenitors in the neonatal brain under pathological conditions $[10,14-16,18,21,30]$. This is particularly true with regard to WMI of the neonatal brain, 


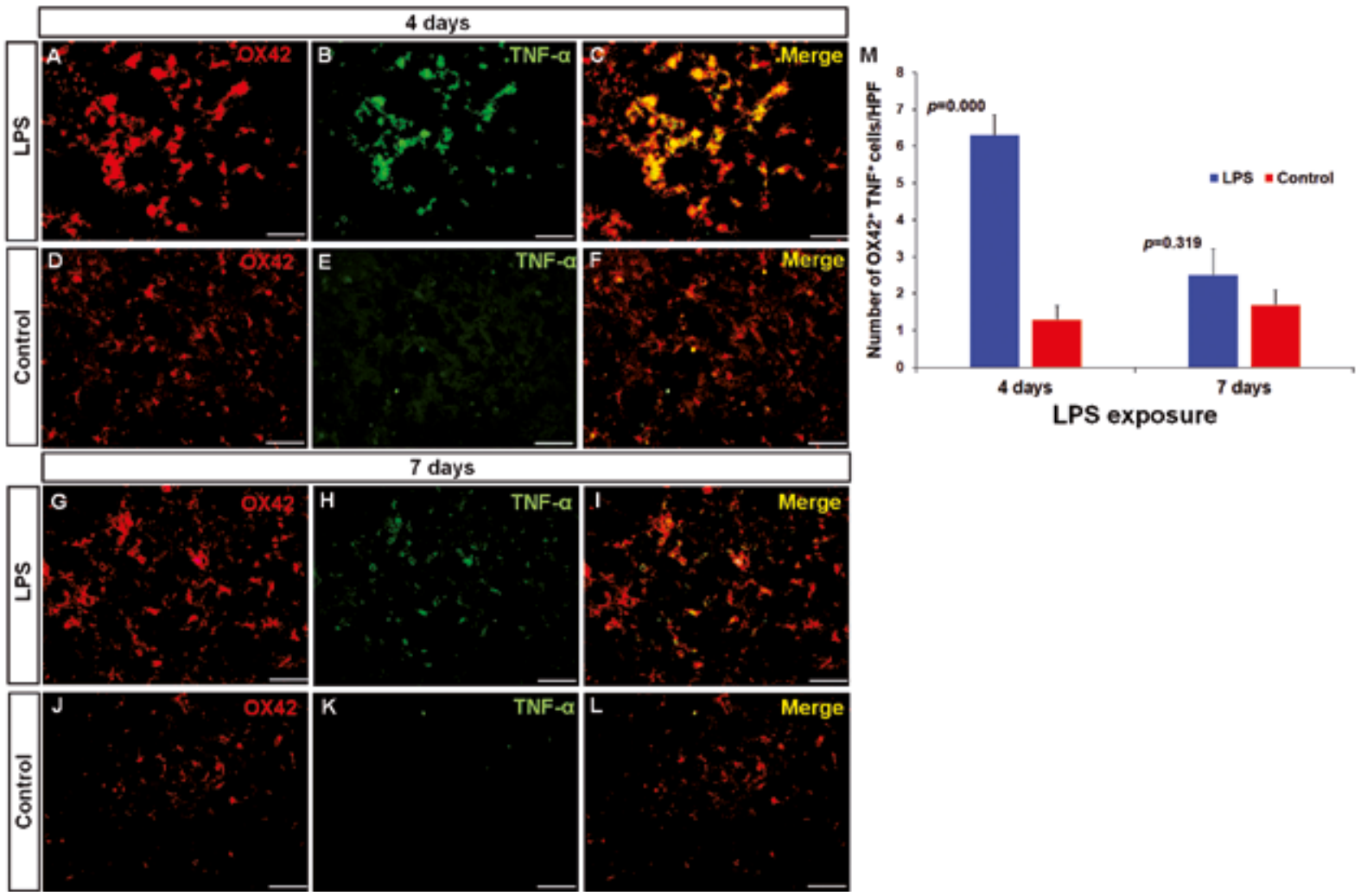

Fig. 3. Double-staining with anti-TNF- $\alpha$ and anti-OX42. Images show that at 4 and 7 days post-LPS exposure, forebrain slices exhibit an increase in the number of cells double-labelled for OX42 and TNF- $\alpha$ (A-L). LPS also increased the number of OX42-positive microglia and their processes (A, G). OX42-positive microglia (red) appear yellowish in colour due to co-localization with TNF- $\alpha$ (green) (C, I) 4 and 7 days after LPS exposure. Relatively few OX42-positive cells can be seen to express TNF- $\alpha$ in control forebrain slices (F). Scale bars, $100 \mu \mathrm{m}$. Numbers of OX42-positive microglia merged with TNF- $\alpha$ per HPF in the forebrain slices were only significantly higher than those in the controls at 4 days after the LPS exposure $(p<0.05)(M)$.

where activated microglia have been reported to release TNF- $\alpha$ and - to a lesser extent - IL-1 $\beta$ during early stages of white matter damage $[16,22,30]$. Interestingly, an in vitro study of inflammatory injury to preOLs revealed that LPS induces selective preOL death by indirectly activating microglia [16]. Moreover, another investigation reported that the overexpression of TNF- $\alpha$ in astrocytes results in OL apoptosis and demyelination [1].

The results of the present study demonstrated a marked increase in TNF- $\alpha$ levels in the culture media of organotypic forebrain slices at 4 days following LPS exposure. Further, activated astrocytes and microglia displayed concurrent increases in the expression of TNF- $\alpha$ coinciding with a marked $\mathrm{OL}$ degeneration. These findings suggest that the activation of microglia and astrocytes might be related to the LPS-induced death of OL progenitors, and that this cascade is perhaps mediated by TNF- $\alpha$ during the early stages of LPS-mediated injury $[10,15,16,20,22,30]$. At 7 days after the LPS exposure, many astrocytes and microglia were still activated by TNF- $\alpha$, and OL progenitors appeared to be in an immature or under-differentiated state; as this stage likely marks the regenerative phase of $\mathrm{OL}$ progenitors, our results suggest that glial cells activated by TNF- $\alpha$ are involved in the maturation of OL progenitors during recovery from injury.

Though experimental studies have reported that glial cells expressing TNF- $\alpha$ might be involved in recovery after injury $[1,17]$, the mechanisms by which astrocytes and microglia contribute to the death or arrested maturation of OL progenitors are not well understood. The aforementioned study 

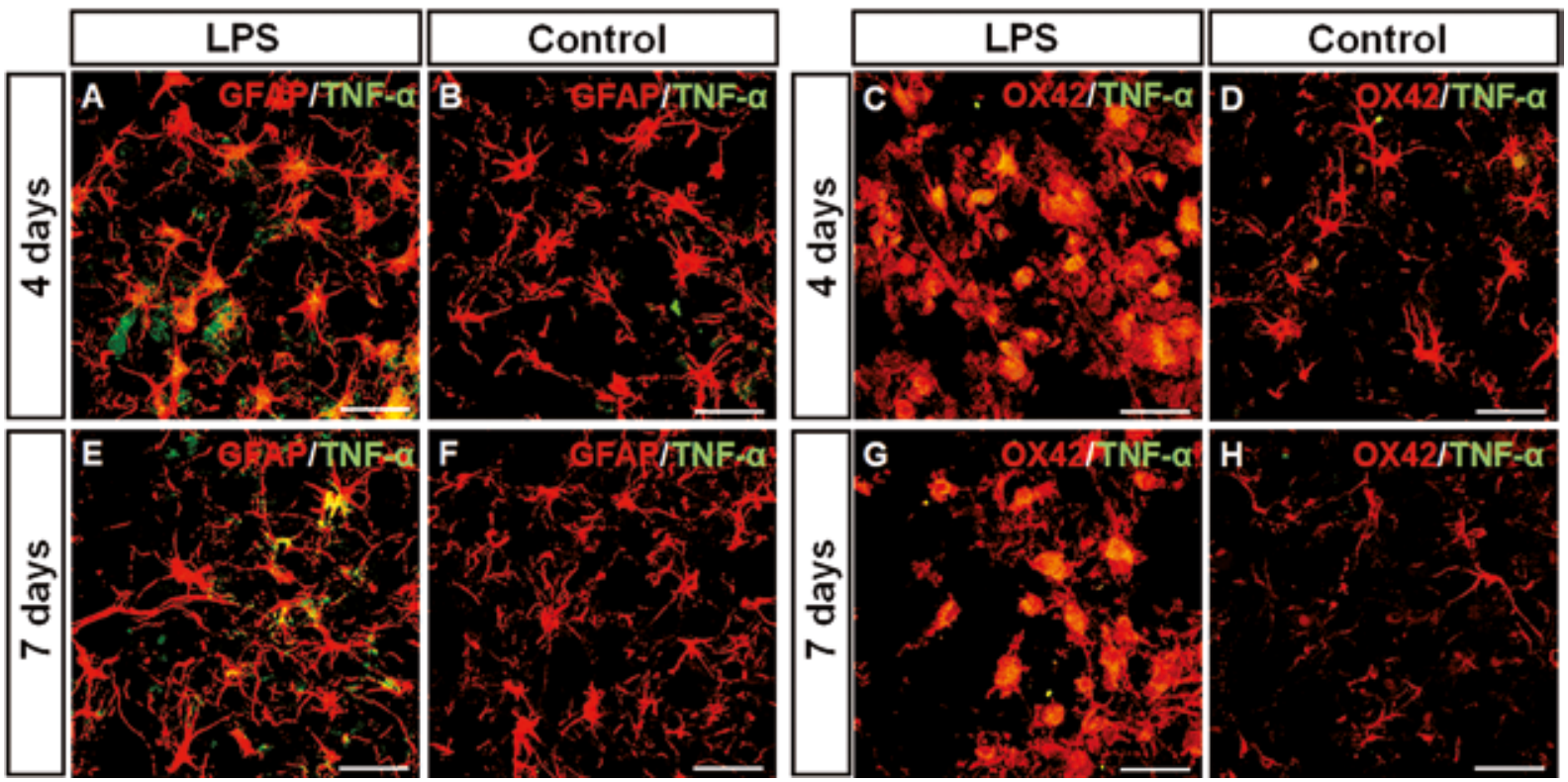

Fig. 4. Confocal laser microscopy findings for double-staining with anti-TNF- $\alpha /$ anti-GFAP and anti-TNF- $\alpha /$ anti-OX42. Confocal laser microscopy revealing detailed GFAP-positive astrocytes and OX42-positive microglia morphologies. Images show that at 4 and 7 days after LPS exposure, GFAP-positive and OX42-positive cells exhibited many well-differentiated processes and yellow cell bodies due to co-localization with TNF- $\alpha$ $(A, C, E, G)$. Cells from control slices, on the other hand, can be seen displaying no co-localization with TNF- $\alpha$ (B, D, F, H). Scale bars, $100 \mu \mathrm{m}$.

reported that TNF $\alpha$ signalling via TNFR1/2 is essential to LPS-triggered preOL death in tissue containing all major glial cell types [16]. Another investigation reported that astrocytes play an essential role in TNF toxicity to preOLs in an environment where all glia cells coexist [15]. In addition, recent evidence has implicated the opening of astrocytic gap junction hemichannels as a key regulating event alongside excessive microglial activation in spreading injury after brain ischemia [18].

Regarding the inhibition of $\mathrm{OL}$ progenitor differentiation by glial cells, it has been reported that a high molecular weight form of hyaluronan (synthesized by astrocytes) may prevent maturation of $\mathrm{OL}$ progenitors in chronic, demyelinated white matter lesions; thus substantially contributing to remyelination failure $[7,9]$. A recent study reported that reactive astrocytes release TNF- $\alpha$ to inhibit the survival of OL progenitors, preventing them from differentiating into mature OLs after spinal cord injury [30]. Moreover, a study modelling toxin-induced demyelination found that TNF is required for preOL proliferation and remyelination: a beneficial effect mediated by
TNF receptor 2 [17]. Another investigation using primary cultures of OL progenitor cells from neonatal rats showed that LPS-activated microglia induced delayed cell death $48 \mathrm{~h}$ after treatment and impair OL development [20]. Other studies have reported that microglia play a dual role in CNS injury, exacerbating damage in some instances and promoting repair or regeneration in others [8,31]. However, we were unable to identify the exact roles of glial cells and cytokines (TNF- $\alpha$ ) in the degeneration, repair or differentiation of OL progenitors in any of the various stages of the injury.

Several human and experimental studies have suggested that the mechanism of myelination failure in perinatal WMI might comprise a combination of degenerative, regenerative, and arrested maturational processes of preOLs $[2,7,9,28,34]$. Similar to previous reports, the results of this in vitro study suggest that maturation arrest or under-differentiation of OL progenitors might play an important role in LPS-induced hypomyelination, as well as in the degeneration or death of preOLs $[6,7,9,21,28]$. We also speculate that inflammation-activated microg- 


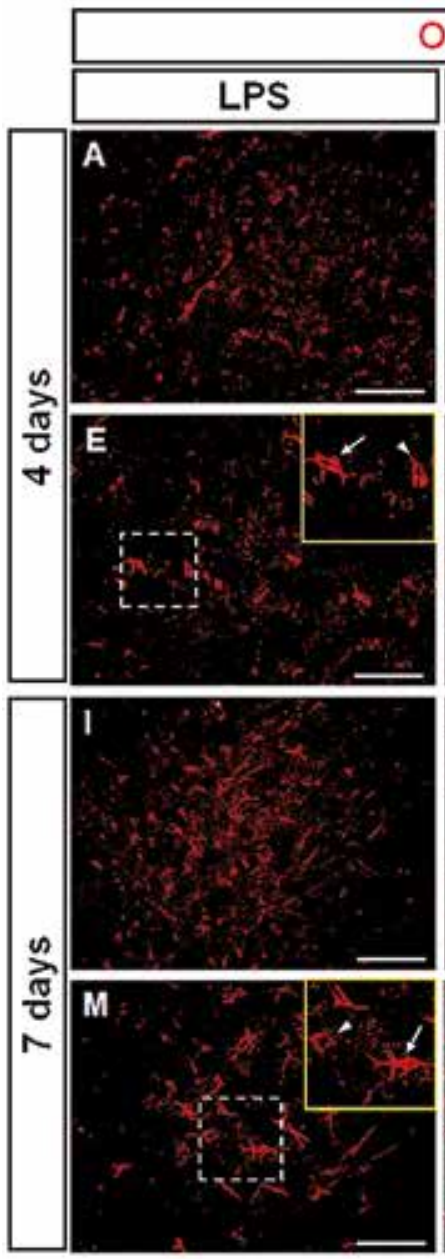

04

Control
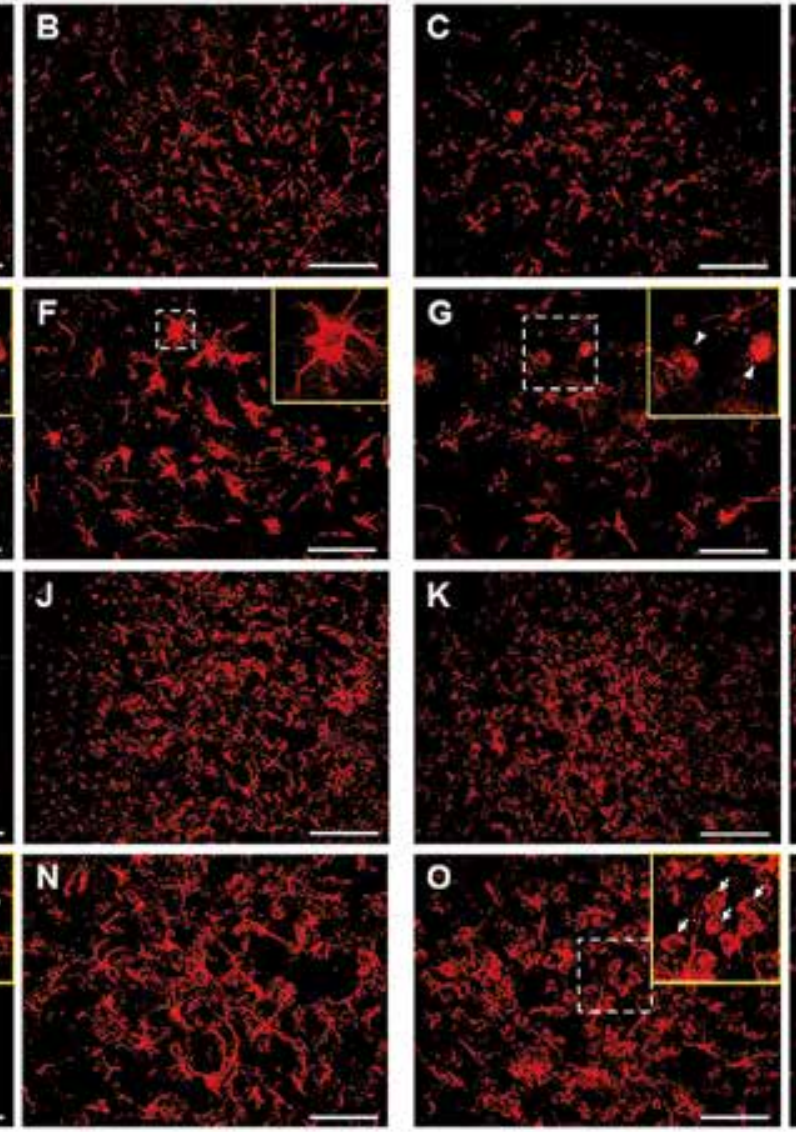
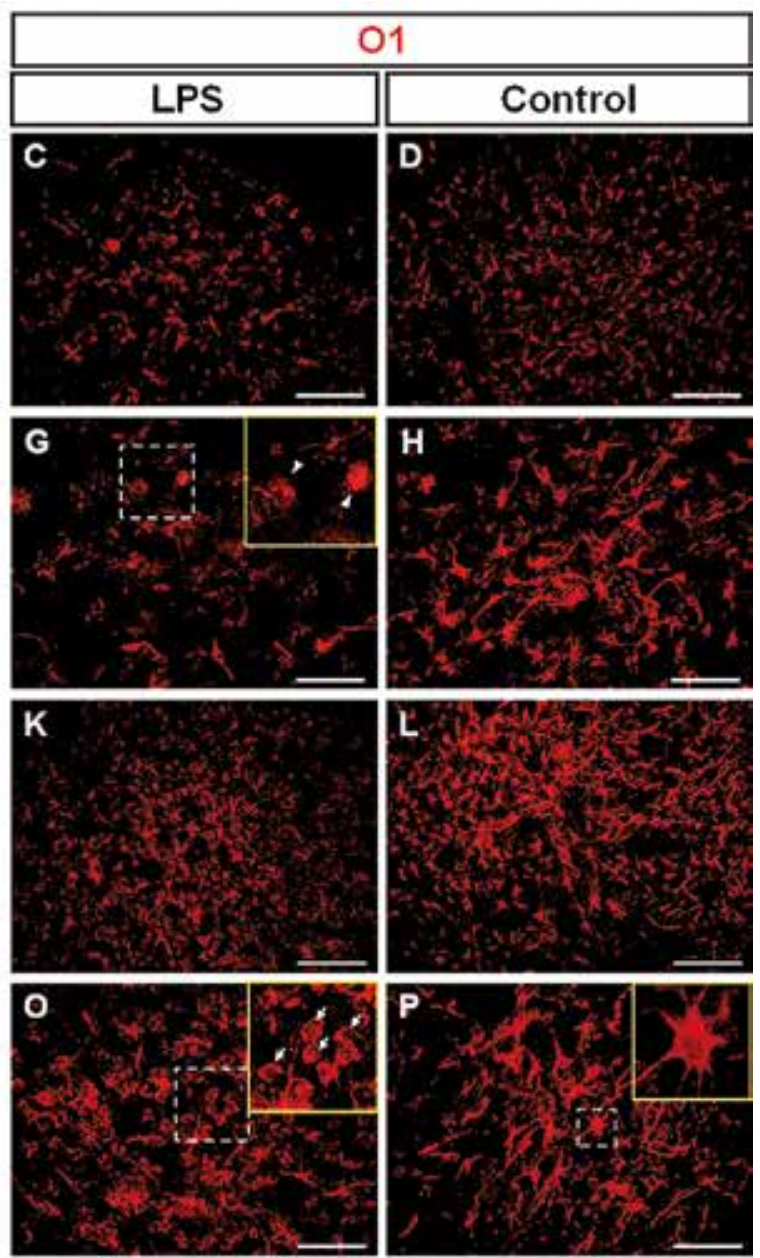
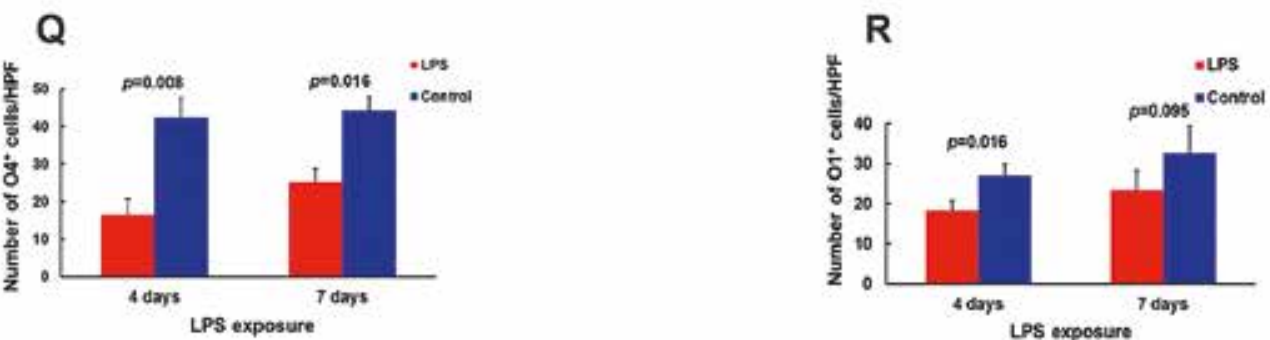

Fig. 5. Immunofluorescent staining for $\mathrm{O} 4$ and 01 . Images showing that at 4 days after exposure, LPS reduced the expression of 04-positive and 01-positive cells in forebrain slices compared to controls (A-D). High power field (HPF) showing 04-positive and 01-positive cells in LPS-exposed forebrain slices with scanty cell bodies and fewer, thinner, more fragmented processes. Some cells also exhibited elongated cell bodies with bipolar or multipolar short processes (E, G). Magnified images (yellow box) showing more detailed morphologies of O4-positive and O1-positive cells. Cytoplasmic condensation and fragmented short processes are visible (arrowheads), as is a cell with an elongated cell body and multipolar processes (arrow). By 7 days following LPS exposure, the expression of O4-positive and O1-positive cells in LPS-exposed slices appears to have increased (I, K). HPF and magnified images showing O4-positive cells with elongated cell bodies and relatively short multipolar processes (arrow). HPF and magnified images also show 04-positive cells with scanty cell bodies and short fragmented processes in LPS-exposed slices (arrowhead) (M). O1-positive cells at 7 days after LPS exposure are shown to have typical, rounded shapes with few processes, suggesting under-differentiation (short arrows) (0). In contrast, O4-positive and O1-positive cells in controls can be seen with preserved, well-differentiated morphologies $(\mathrm{F}, \mathrm{H}, \mathrm{N}, \mathrm{P})$. The number of O4-positive cells in LPS-exposed slices (both at 4 and 7 days post-application) was significantly higher than in the controls $(p<0.05)$; however, the number of 01-positive cells was only significantly higher relative to controls at 4 days after LPS exposure $(p<0.05)(\mathbf{Q}, \mathbf{R})$. Scale bars, A-D and I-L, $200 \mu \mathrm{m}, \mathbf{E}-\mathbf{H}$ and $\mathbf{M}-\mathbf{P}, 100 \mu \mathrm{m}$. 

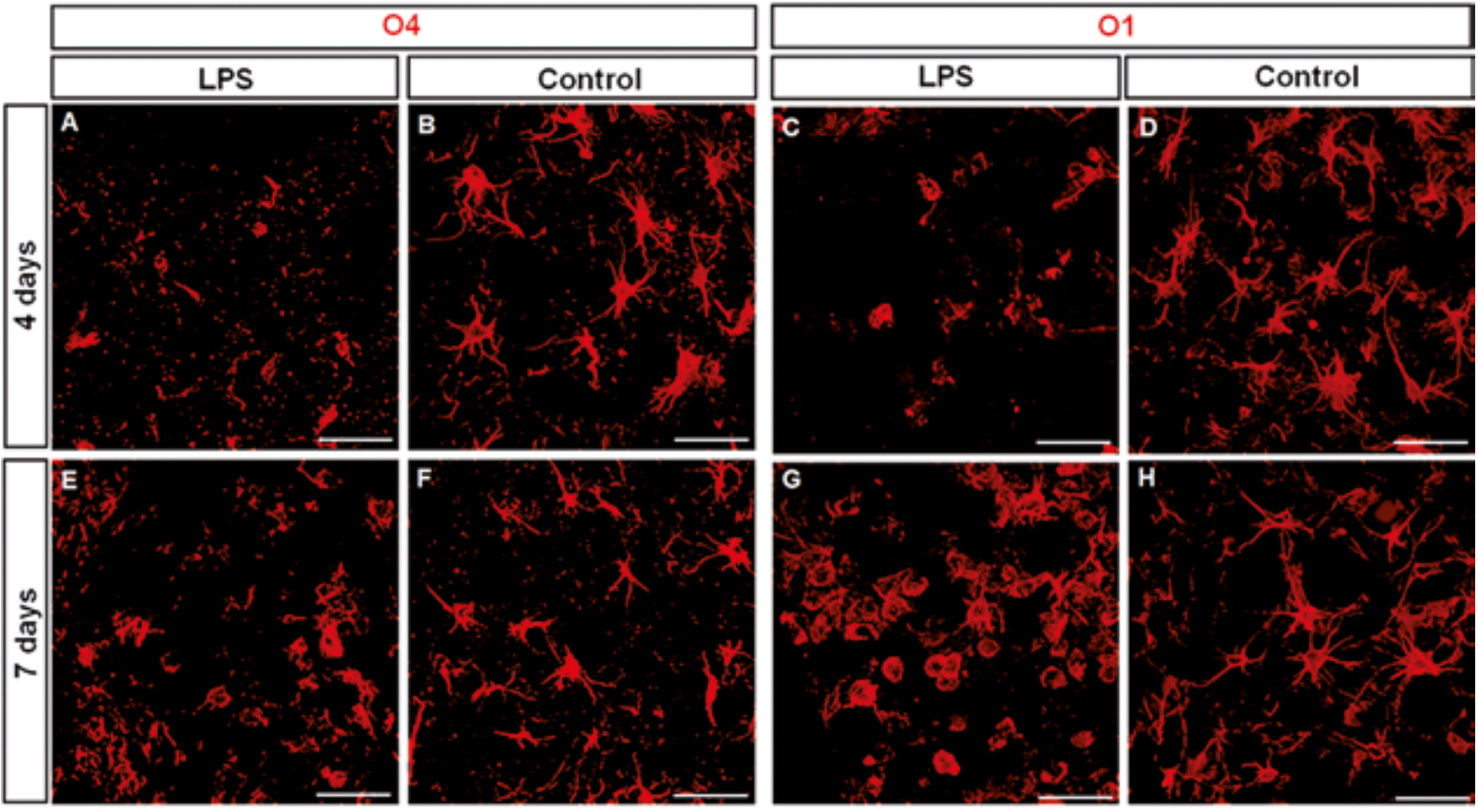

Fig. 6. Confocal laser microscopic findings of O4-positive and O1-positive cells. At 7 days after LPS, degenerated O4-positive cells can still be visualized; however, some 04-positive and many 01-positive cells exhibit typical round bodies with few short processes, suggesting under-differentiated or immature states (E, G). In contrast, well-differentiated O4-positive and O1-positive cells can be seen in control forebrain slices (B, D, F, H). Scale bars, $100 \mu \mathrm{m}$.
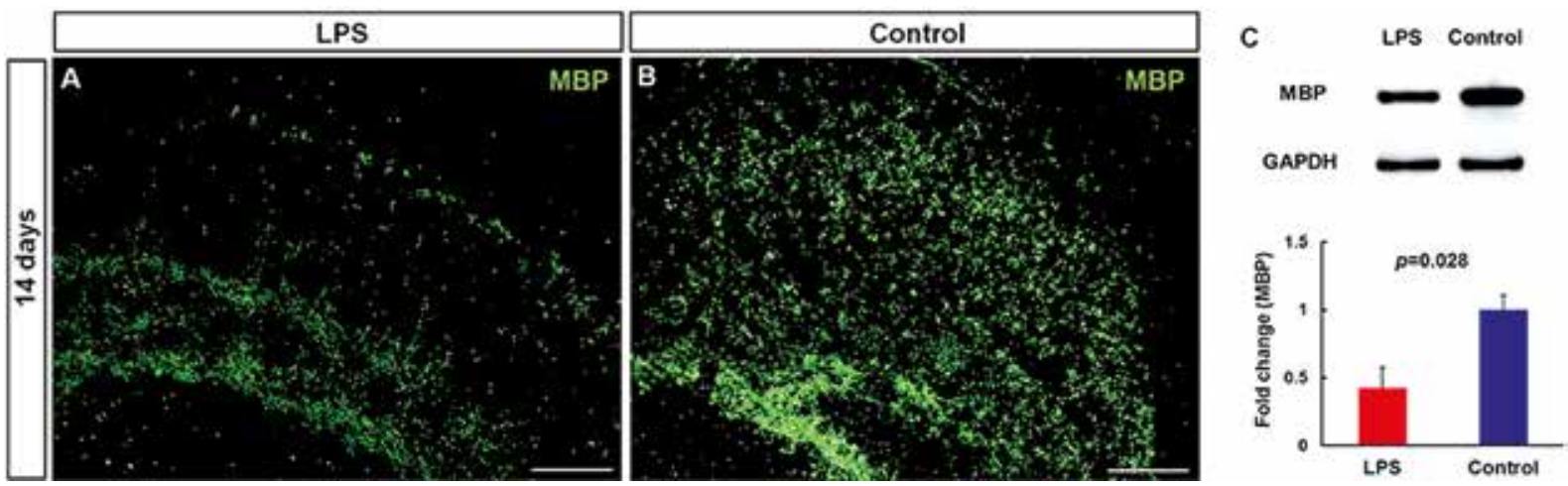

Fig. 7. Immunofluorescent staining and western blotting for myelin basic protein (MBP). Compared to controls, LPS reduced the immunofluorescent expression of MBP in forebrain slices 14 days after exposure (A, B). Western blotting conducted 14 days after exposure demonstrated that LPS significantly reduced the expression of MBP when compared to controls $(n=4)(p<0.05)(C)$. Scale bars, $1 \mathrm{~mm}$.

lia and astrocytes expressing TNF- $\alpha$ might contribute to myelination failure. Further research is needed to evaluate the mechanism of glial cell involvement, the interaction among glial cells, and the role of cytokines in LPS-induced hypomyelination of premature brains.

\section{Acknowledgements}

This work was supported by the Ministry of Education of the Republic of Korea and the National Research Foundation of Korea (NRF2012R1A1A1011783, NRF-2015R1D1A1A01059014, and NRF-2018R1D1A1A02047523). 


\section{Disclosure}

\section{The authors report no conflict of interest.}

\section{References}

1. Akassoglou K, Bauer J, Kassiotis G, Pasparakis M, Lassmann H, Kollias G, Probert L. Oligodendrocyte apoptosis and primary demyelination induced by local TNF/p55TNF receptor signaling in the central nervous system of transgenic mice: models for multiple sclerosis with primary oligodendrogliopathy. Am J Pathol 1998; 153: 801-813.

2. Back SA. Brain injury in the preterm infant: new horizons for pathogenesis and prevention. Pediat Neurol 2015; 53: 185-192.

3. Back SA, Luo NL, Borenstein NS, Levine JM, Volpe JJ, Kinney HC. Late oligodendrocyte progenitors coincide with the developmental window of vulnerability for human perinatal white matter injury. J Neurosci 2001; 21: 1302-1312.

4. Back SA, Miller SP. Brain injury in premature neonates: A primary cerebral dysmaturation disorder? Ann Neurol 2014; 75: 469-486.

5. Back SA, Riddle A, McClure MM. Maturation-dependent vulnerability of perinatal white matter in premature birth. Stroke 2007; 38: 724-730.

6. Billiards SS, Haynes RL, Folkerth RD, Borenstein NS, Trachtenberg FL, Rowitch DH, Ligon KL, Volpe JJ, Kinney HC. Myelin abnormalities without oligodendrocyte loss in periventricular leukomalacia. Brain Pathol 2008; 18: 153-163.

7. Buser JR, Maire J, Riddle A, Gong X, Nguyen T, Nelson K, Luo NL, Ren J, Struve J, Sherman LS, Miller SP, Chau V, Hendson G, Ballabh P, Grafe MR, Back SA. Arrested preoligodendrocyte maturation contributes to myelination failure in premature infants. Ann Neurol 2012; 71: 93-109.

8. Butovsky O, Landa G, Kunis G, Ziv Y, Avidan H, Greenberg N, Schwartz A, Smirnov I, Pollack A, Jung S, Schwartz M. Induction and blockage of oligodendrogenesis by differently activated microglia in an animal model of multiple sclerosis. J Clin Invest 2006; 116: 905-915.

9. Dean JM, Riddle A, Maire J, Hansen KD, Preston M, Barnes AP, Sherman LS, Back SA. An organotypic slice culture model of chronic white matter injury with maturation arrest of oligodendrocyte progenitors. Mol Neurodegener 2011; 6: 46.

10. Deng Y, Xie D, Fang M, Zhu G, Chen C, Zeng H, Lu J, Charanjit K. Astrocyte-derived proinflammatory cytokines induce hypomyelination in the periventricular white matter in the hypoxic neonatal brain. PLoS One 2014; 9:e87420.

11. Deverman BE, Patterson PH. Cytokines and CNS Development. Neuron 2009; 64: 61-78.

12. Fields RD, Stevens-Graham B. New insights into neuron-glia communication. Science 2002; 298: 556-562.

13. Fischer R, Wajant H, Kontermann R, Pfizenmaier K, Maier O. Astrocyte-Specific Activation of TNFR2 Promotes Oligodendrocyte Maturation by Secretion of Leukemia Inhibitory Factor. Glia 2014; 62: 272-283.

14. Kadhim H, Tabarki B, Verellen G, De Prez C, Rona AM, Sebire G. Inflammatory cytokines in the pathogenesis of periventricular leukomalacia. Neurology 2001; 56: 1278-1284.
15. Kim S, Steelman AJ, Koito H, Li J. Astrocytes promote TNF-mediated toxicity to oligodendrocyte precursors. J Neurochem 2011; 116: 53-66.

16. Li J, Ramenaden ER, Peng J, Koito H, Volpe JJ, Rosenberg PA. Tumor necrosis factor alpha mediates lipopolysaccharide-induced microglial toxicity to developing oligodendrocytes when astrocytes are present. J Neurosci 2008; 28: 5321-5330.

17. Maier O, Fischer R, Agresti C, Pfizenmaier K. TNF receptor 2 protects oligodendrocyte progenitor cells against oxidative stress. Biochem Biophys Res Commun 2013; 440: 336-341.

18. Mallard C, Davidson JO, Tan S, Green CR, Bennet L, Robertson NJ, Gunn AJ. Astrocytes and microglia in acute cerebral injury underlying cerebral palsy associated with preterm birth. Pediatr Res 2014; 75: 234-240.

19. Miron VE, Kuhlmann T, Antel JP. Cells of the oligodendroglial lineage, myelination, and remyelination. Biochim Biophys Acta 2011; 1812: 184-193.

20. Pang Y, Cai Z, Rhodes PG. Effects of lipopolysaccharide on oligodendrocyte progenitor cells are mediated by astrocytes and microglia. J Neurosci Res 2000; 62: 510-520.

21. Pang Y, Campbell L, Zheng B, Fan L, Cai Z, Rhodes P. Lipopolysaccharide-Activated microglia induce death of oligodendrocyte progenitor cells and impede their development. Neuroscience 2010; 166: 464-475.

22. Pang Y, Fan LW, Tien LT, Dai X, Zheng B, Cai Z, Lin RC, Bhatt A. Differential roles of astrocyte and microglia in supporting oligodendrocyte development and myelination in vitro. Brain Behav 2013; 3: 503-514.

23. Parikh NA, Pierson CR, Rusin JA. Neuropathology associated with diffuse excessive high signal intensity abnormalities on magnetic resonance imaging in very preterm infants. Pediatr Neurol 2016; 65: 78-85.

24. Pfeiffer SE, Warrington AE, Bansal R. The oligodendrocyte and its many cellular processes. Trends Cell Biol 1993; 3: 191-197.

25. Pleasure D, Soulika A, Singh SK, Gallo V, Bannerman P. Inflammation in white matter: clinical and pathophysiological aspects. Ment Retard Dev Disabil Res Rev 2006; 12: 141-146.

26. Riddle A, Dean J, Buser JR, Gong X, Maire J, Chen K, Ahmad T, Cai V, Nguyen T, Kroenke CD, Hohimer AR, Back SA. Histopathological correlates of magnetic resonance imaging-defined chronic perinatal white matter injury. Ann Neurol 2011; 70 : 493-507.

27. Romero J, Muniz J, Logica Tornatore T, Holubiec M, Gonzalez J, Barreto GE, Guelman L, Lillig CH, Blanco E, Capani F. Dual role of astrocytes in perinatal asphyxia injury and neuroprotection. Neurosci Lett 2014; 565: 42-46.

28. Segovia KN, McClure M, Moravec M, Luo NL, Wan Y, Gong X, Riddle A, Craig A, Struve J, Sherman LS, Back SA. Arrested oligodendrocyte lineage maturation in chronic perinatal white matter injury. Ann Neurol 2008; 63: 520-530.

29. Stoppini L, Buchs PA, Muller D. A simple method for organotypic cultures of nervous tissue. J Neurosci Methods 1991; 37: 173-182.

30. Su Z, Yuan Y, Chen J, Zhu Y, Qiu Y, Zhu F, Huang A, He C. Reactive astrocytes inhibit the survival and differentiation of oligodendrocyte precursor cells by secreted TNF-alpha. J Neurotrauma 2011; 28: 1089-1100. 
31. Taylor DL, Pirianov G, Holland S, McGinnity CJ, Norman AL, Reali C, Diemel LT, Gveric D, Yeung D, Mehmet H. Attenuation of proliferation in oligodendrocyte precursor cells by activated microglia. J Neurosci Res 2010; 88: 1632-1644.

32. Vilhardt F. Microglia: phagocyte and glia cell. Int J Biochem Cell Biol 2005; 37: 17-21.

33. Volpe JJ. Neurobiology of periventricular leukomalacia in the premature infant. Pediatr Res 2001; 50: 553-562.

34. Volpe JJ. Brain injury in premature infants: a complex amalgam of destructive and developmental disturbances. Lancet Neurol 2009; 8: 110-124.

35. Volpe JJ. The encephalopathy of prematurity-brain injury and impaired brain development inextricably intertwined. Semin Pediatr Neurol 2009; 16: 167-178. 